\title{
BMJ Open The UK Cardiac and Vascular Surgery Interventional Anaemia Response (CAVIAR) Study: protocol for an observational cohort study to determine the impact and effect of preoperative anaemia management in cardiac and vascular surgical patients
}

\author{
Marisa Chau, ${ }^{1}$ Toby Richards, ${ }^{1}$ Caroline Evans, ${ }^{2}$ Anna Butcher, ${ }^{1}$ Timothy Collier, ${ }^{3}$ \\ Andrew Klein ${ }^{4}$
}

To cite: Chau $\mathrm{M}$,

Richards T, Evans C, et al. The UK Cardiac and Vascular Surgery Interventional Anaemia Response (CAVIAR) Study: protocol for an observational cohort study to determine the impact and effect of preoperative anaemia management in cardiac and vascular surgical patients. BMJ Open 2017;7:e014872. doi:10.1136/ bmjopen-2016-014872

- Prepublication history and additional material is available. To view please visit the journal (http://dx.doi.org/10.1136/ bmjopen-2016-014872).

Received 23 0ctober 2016 Revised 28 February 2017 Accepted 9 March 2017

CrossMark

For numbered affiliations see end of article.

Correspondence to

Marisa Chau; m.chau@ucl.ac.uk

\section{ABSTRACT}

Introduction Preoperative anaemia is linked to poor postsurgical outcome, longer hospital stays, greater risk of complications and mortality. Currently in the UK, some sites have developed anaemia clinics or pathways that use intravenous iron to correct iron deficiency anaemia prior to surgery as their standard of care. Although intravenous iron has been observed to be effective in a variety of patient settings, there is insufficient evidence in its use in cardiac and vascular patients. The aim of this study is to observe the impact and effect of anaemia and its management in patients undergoing cardiac and vascular surgery. In addition, the UK Cardiac and Vascular Surgery Interventional Anaemia Response (CAVIAR) Study is also a feasibility study with the aim to establish anaemia management pathways in the preoperative setting to inform the design of future randomised controlled trials.

Methods and analysis The UK CAVIAR Study is a multicentre, stepped, observational study, in patients awaiting major cardiac or vascular surgery. We will be examining different haematological variables (especially hepcidin), functional capacity and patient outcome. Patients will be compared based on their anaemia status, whether they received intravenous iron in accordance to their hospital's preoperative pathway, and their disease group. The primary outcomes are the change in haemoglobin levels from baseline (before treatment) to before surgery; and the number of successful patients recruited and consented (feasibility). The secondary outcomes will include changes in biomarkers of iron deficiency, length of stay, quality of life and postoperative recovery.

Ethics and dissemination The study protocol was approved by the London-Westminster Research Ethics Committee (15/L0/1569, 27 November 2015). NHS approval was also obtained with each hospital trust. The findings of the study will be published in peer-reviewed journals.

Trial registration number Clinical Trials registry (NCT02637102) and the ISRCTN registry (ISRCTN55032357).
Strengths and limitations of this study

This is the first study examining how iron treatment and major surgery affects haematological variables, functional capacity and patient outcome.

- Furthermore, this study will also be the first to compare cardiac with vascular patient groups.

- The results of this study will provide information in the understanding of preoperative anaemia, and whether correcting iron deficiency anaemia will lead to improved surgical outcomes.

- Slight variations in preoperative anaemia pathways used in different hospitals.

- Different intravenous iron will be used in different hospitals.

\section{INTRODUCTION}

There is increasing evidence that preoperative anaemia is associated with a higher risk of complications, prolonged hospital stay, transfusion requirement and mortality. ${ }^{1-5}$ Anaemia is becoming increasingly prevalent largely due to an ageing population. The estimated prevalence of anaemia is up to $50 \%$ of patients in some hospitals. ${ }^{6}$ There is also considerable variability in prevalence in different hospitals and regions of the UK. ${ }^{7}$ Nutritional deficiencies of iron, folate and/ or vitamin $\mathrm{B}_{12}$ contribute to approximately a third of the cases preoperatively, whereas functional iron deficiency (FID) or iron restriction appears to be more common but its relevance is less well understood.

FID exists when there is failure of the bioavailability of iron for erythrogenesis. The mechanism occurs via inflammatory 
mediators induced by pre-existing illness or comorbid disease. Hepcidin, an important regulator of iron homoeostasis, is upregulated under inflammatory conditions ${ }^{9}$ and prevents export of iron from cells. This results in the increased uptake and retention of iron from the circulation by the reticular endothelial system, thus reducing bioavailability of iron for erythrogenesis. ${ }^{10}$ A retrospective study by Hung and colleagues ${ }^{8}$ demonstrated an association of elevated hepcidin with poorer outcome in cardiac patients. Therefore strategies in correcting and managing FID may play a vital role in improving postsurgical outcomes.

Intravenous iron has been shown to be an effective treatment of anaemia in a variety of settings, including heart failure, inflammatory bowel disease and orthopaedic surgery. ${ }^{11-14}$ The Cochrane review by Gurusamy and colleagues ${ }^{15}$ examined the effectiveness of different iron therapies in patients with anaemia and concluded that due to significant heterogeneity and low quality of the trials reviewed, there were insufficient grounds to support the role of treating patients with intravenous iron preoperatively. The updated review however showed increased efficacy of intravenous iron in correcting haemoglobin levels $(\mathrm{Hb})^{16}$ as well as treatment with intravenous iron showed improvement in symptoms, exercise capacity and quality of life, typically in patients with heart failure. ${ }^{11} 14$ There is still insufficient evidence for the effectiveness it may have in cardiac patients. ${ }^{17}$

National guidelines have recommended that anaemia should be identified before surgery and treated if appropriate. ${ }^{18}$ In the UK, a number of centres have introduced anaemia clinics or developed preoperative anaemia pathways incorporating intravenous iron as part of their standard of care. As the current evidence for different iron therapies is limited and variation exists, it is important to understand how these treatments given preoperatively may affect patient fitness for surgery and outcomes.

In light of this, we have designed a study to observe the impact and effect of anaemia and its management in patients undergoing cardiac and vascular surgery. We also aim to assess the regional variations in preoperative pathways, patients' outcomes postoperatively and their requirement for transfusion. In addition, this study aims to gather information about the feasibility of establishing anaemia management pathways in the preoperative setting to guide the design of future randomised controlled trials (RCTs).

\section{METHODS AND ANALYSIS}

This study is a multicentre, stepped, prospective, observational platform comprising two cohorts in patients awaiting major cardiac or vascular surgery. In the first cohort we will examine the effect of anaemia and outcomes (cohort 1), and in the second cohort we will assess the effect of treatment of anaemia (cohort 2) in both population groups. The study is stepped, whereby cohort 1 will initially be identified on the day of surgery and then following feasibility at least 10 days before operation. This enables a step to cohort 2 as the sites can feasibly identify and recruit patients in the appropriate time frame. The advantage of this is that the study can examine a number of outcome variables, and will permit calculation of the effect of each variable on the probability of developing the outcome of interest (relative risk) (please see online supplementary file for the STROBE checklist) ${ }^{19}$

Patients will be identified through routine clinical care, which, depending on the participating site, may include surgical outpatients, preadmission clinics, or referral for cardiopulmonary exercise test (CPET) or 6 min walk test (6MWT) prior to their planned surgery. Local clinicians or research nurses will screen scheduled elective surgery lists and their haemoglobin blood results (within the last 3 months) for eligible patients. Frequency of the screening and contact with the patient has been left to the discretion of the local staff as time and resources vary between hospitals. In addition, each site will be requested to recruit patients in a consecutive manner. The study therefore acts to be a feasibility assessment of local teams to identify patients for preoperative clinical trials.

Anaemia in this study satisfies WHO criteria with $(\mathrm{Hb})<130 \mathrm{~g} / \mathrm{L}$ in men and $<120 \mathrm{~g} / \mathrm{L}$ in women. ${ }^{20}{ }^{21}$

Inclusion criteria are: patients aged 18 years or over, undergoing elective cardiac or vascular surgery (eg, coronary artery bypass graft (CABG), valve surgery, repair or replacement of thoracic or abdominal aorta (open or endovascular), revascularisation or endarterectomy), with written informed consent.

Exclusion criteria are: patients who are pregnant or lactating, undergoing renal dialysis (current or planned within the next 12 months), prisoners and patients who have an underlying history of learning disabilities or adults who do not have mental capacity to consent for themselves.

Recruited patients will then be categorised as below (see figure 1).

1. Patients prior to major cardiac or vascular surgery, in whom consent is taken before operation

2. Patients prior to major cardiac or vascular surgery, in whom consent is taken at least 10 or more days before operation

3. Patients prior to major cardiac or vascular surgery, in whom consent is taken at least 10 or more days before operation in whom a functional exercise assessment is performed (CPET or 6MWT)

\section{Substudy arm}

In patients who receive intravenous iron as part of normal clinical practice at that hospital:

1. Patients who have consented for blood sampling (ie, additional core blood samples) before and after intravenous iron

2. Patients who have consented for blood sampling and in addition in whom a functional exercise assessment is performed (CPET or 6MWT) before and after intravenous iron 


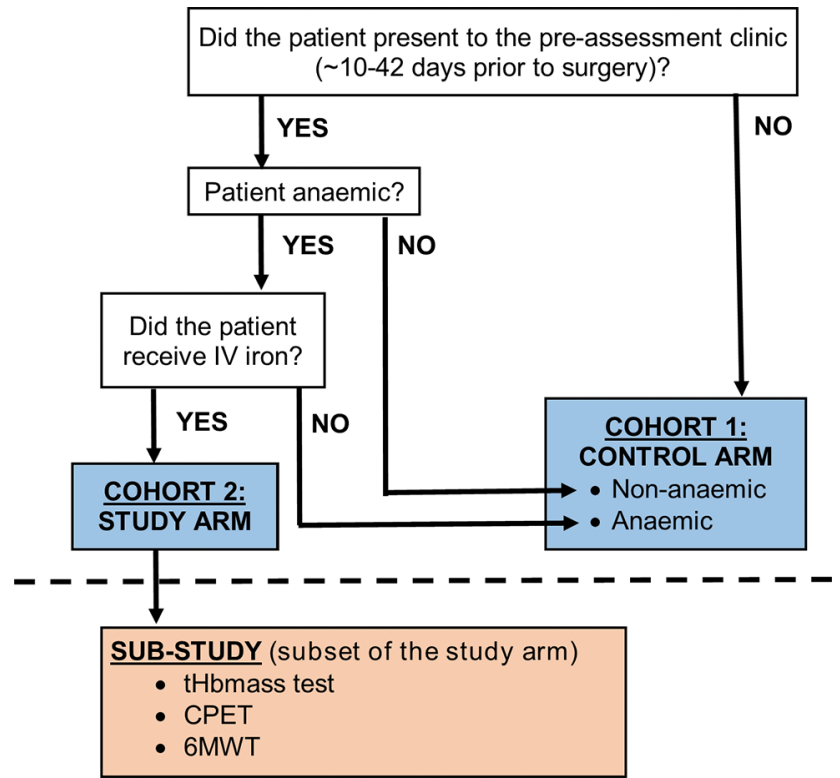

Figure 1 Patient categorisation. 6MWT, 6 mins walk test; CPET, cardiopulmonary exercise test; IV, intravenous; tHbmass, total haemoglobin mass.

3. Patients who have consented for blood sampling and in addition in whom a total haemoglobin mass (tHbmass) test is performed before and after intravenous iron

\section{Assessments}

Patient data will be obtained from medical records and will be used to assess the approach to anaemia management between the participating sites. Information will include patients' past medical history, medications, bloods (including haematinics, ferritin and $\mathrm{C}$ reactive protein), CPET or 6MWT (if applicable), quantity of blood transfused, length of intensive care unit (ICU) and hospital stay and complications relating to index surgery. Data will be collected from consent to prospectively during hospital admission, up until 4-6 weeks at routine clinic follow-up appointment.

In addition to the routine medical information, the trial will also request additional assessments (see online supplementary file 1). These include:

1. Additional core blood tests to examine iron status, hepcidin and serum proteins;

2. Health-related Quality of life (HRQoL) questionnaires (comprised of Single Question Outcome Measure, Multidimensional Fatigue Inventory and EuroQol in the study arm only;

3. Days alive and out of hospital (DAOH) at follow-up for all arms (at 30 days postsurgery), calculated as per Ariti and colleagues. ${ }^{82}$

Substudy only:

1. If CPET/6MWT was done routinely at baseline before iron therapy (eg, preassessment clinic), we would ask the patient to repeat the same test after iron therapy. Both CPET and 6MWT will be included in this study. CPET is an objective measure in determining response of patients who undergo major surgery through quantifying oxygen consumption and carbon dioxide production. ${ }^{23}$ Although it is considered the gold standard assessment of functional capacity, it is however not routinely used in all NHS hospitals. The alternative option is the 6MWT, which is easy to administer and does not require special equipment, and involves walking up and down a $100 \mathrm{ft}$ hallway. The limitation to the 6MWT is that it is only a good indicator for daily activities and not so much on peak oxygen uptake. ${ }^{24}$

2. Selected sites will conduct the tHbmass test (as this requires special equipment and training) using the optimised carbon monoxide rebreathing method $^{25}$ before and after iron therapy. tHbmass represents absolute mass of circulating haemoglobin and has been suggested to be a more reliable marker in determining oxygen carrying capacity and in predicting postsurgical outcome compared with using $\mathrm{Hb}$ or blood volume alone. ${ }^{26}$ The test involves breathing through a tube that is attached to a bag containing small amounts of carbon monoxide for $2 \mathrm{~min}$. Blood samples will be taken from the fingertip or earlobe before and after carbon monoxide exposure to determine carboxyhaemoglobin concentration. ${ }^{25}$ This will be exploratory in nature to determine the feasibility and practicality if carried out in a 'hospital' setting.

Where possible, assessments will coincide with routine hospital schedules and clinical blood tests. All data will be entered by local staff into a password-protected, electronic case report form (eCRF). Any missing data will be noted in the eCRF. All information collected will be kept confidential and in accordance with the UK Data Protection Act 1998. Figure 2 shows the flow chart of the UK Cardiac and Vascular Surgery Interventional Anaemia Response (CAVIAR) Study.

\section{Patient outcome measures}

The primary outcomes for this study are:

- Change in Hbfrom baseline (before treatment) to before surgery in the study arm; and

- The number of successful patients recruited and consented (feasibility).

The secondary outcomes are:

- Change in $\mathrm{Hb}$ from before treatment to postsurgery;

- Changes in biomarkers of iron deficiency (eg, hepcidin, ferritin, transferrin saturation) from before and after iron therapy,

- Units of blood transfused perioperatively; length of stay (LOS) in hospital and ICU;

- Renal function (change in creatinine) from baseline to before or after surgery; complications during hospital stay;

- HRQoL questionnaires before and after iron therapy (or after surgery for substudy). 


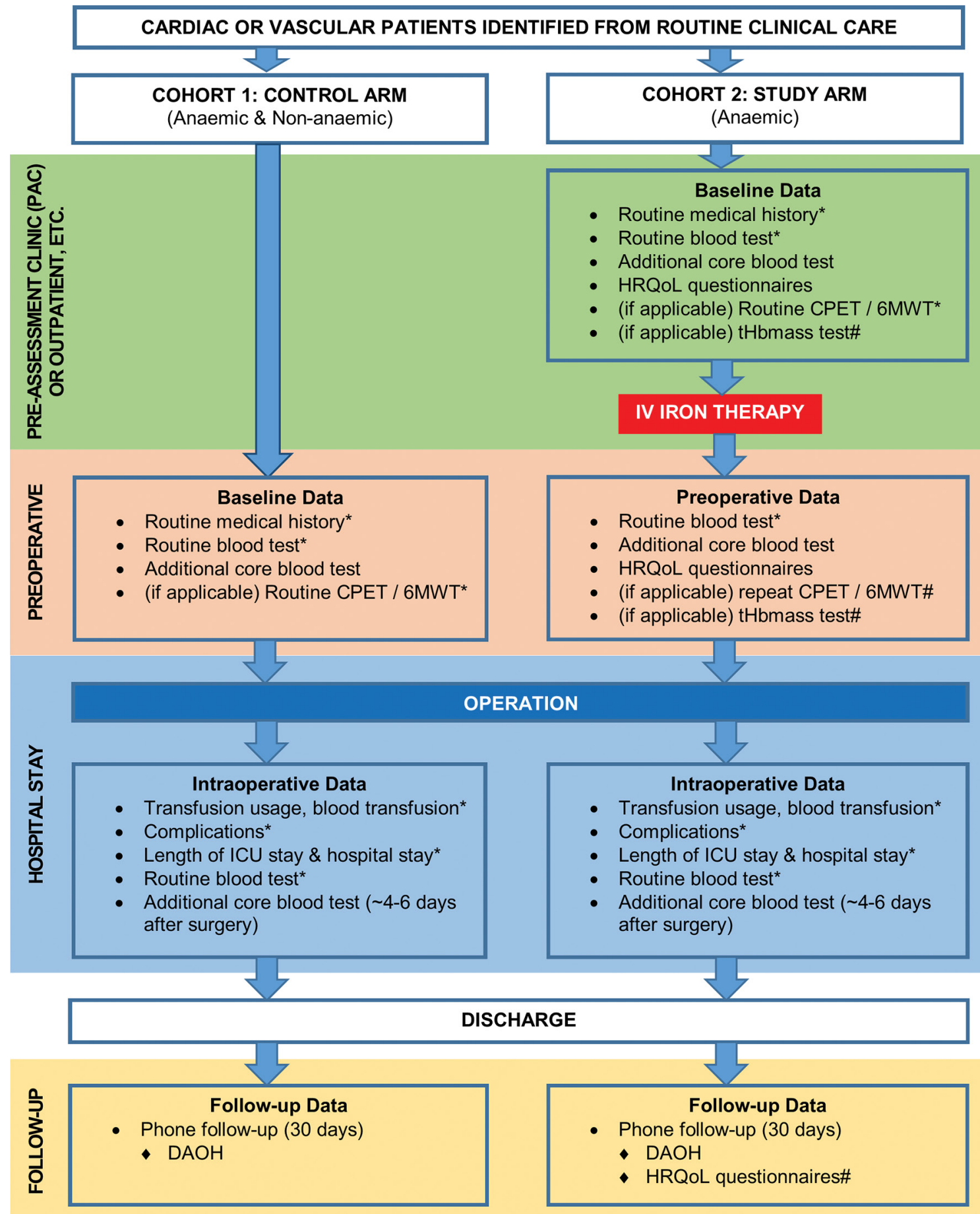

Figure 2 Flow chart of the UK CAVIAR Study. * Collected from patient's medical records. \# Only relevant for the substudy arm. CAVIAR, Cardiac and Vascular Surgery Interventional Anaemia Response; 6MWT, 6 min walking test; CPET, cardiopulmonary exercise test; DAOH, days alive and out of hospital; HRQoL, health-related quality of life; ICU, intensive care unit; IV, intravenous; tHbmass, total haemoglobin mass.

For the substudy only arm: CPET/6 MWT before and after iron therapy; and tHbMass test before and after iron therapy.

\section{Sample size calculation}

The sample size was calculated for the primary end point of change in $\mathrm{Hb}$ from baseline to 10-42 days before surgery for those who receive intravenous iron. Assuming that the SD for $\mathrm{Hb}$ will be $12 \mathrm{~g} / \mathrm{L},{ }^{727}$
72 patients will provide $90 \%$ power at the $5 \%$ significance level (allowing for up to $10 \%$ loss to follow-up) to demonstrate a difference in the change from baseline in $\mathrm{Hb}$ of $10 \mathrm{~g} / \mathrm{L}$. We will therefore recruit 72 cardiac surgery patients and 72 vascular surgery patients for the study arm (cohort 2). In patients who received intravenous iron in the study arm, an arbitrary number of 12 from each of the patient groups will be asked to participate in the substudy arm. This substudy arm will be 
exploratory in nature, used to determine the feasibility and practicality to carry these additional assessments in a 'hospital setting'.

For the control arm, we will recruit patients who are anaemic and non-anaemic (cohort 1). Controls will be collected sequentially for the duration of the trial to avoid time bias and sampling error. Therefore, control patients will be recruited at twice the number than the study group. Overall, a total of 432 patients will be recruited to this study. See online supplementary file 2 for the number of patients per group.

\section{Statistical analysis}

Baseline demographics of the patients will be described using summary statistics. Continuous variables will be summarised using the mean and SD if approximately normally distributed. Continuous variables that are not normally distributed will be summarised using the median and IQR. Differences between groups wil be assessed using two-sample t-tests for normally distributed variables. For variables that are not normally distributed an appropriate transformation will be carried out followed by a two-sample t-test. If no appropriate transformation can be found a non-parametrical test will be used. Categorical variables will be described using frequency and percentage and differences between groups will be tested using $\chi^{2}$ or Fisher's exact tests as appropriate. The linear association between tHbmass, maximal oxygen consumption $\left(\mathrm{Vo}_{2}\right)$ and iron indices will be evaluated using Pearson correlation coefficient and linear regression. Adjusted analyses will be carried out using multivariable linear regression. The accuracy of the subsequent model for outcome predictions will be determined using a receiver operating characteristic (ROC) curve analysis. Depending on the distribution of the data, liner regression (with a $\log$ transformation), Poisson or negative binomial model will be used to assess the value of preoperative tHbmass as a predictor of the primary outcome, postoperative morbidity and secondary outcome variables (hospital LOS, ICU LOS).

\section{DISCUSSION}

Preoperative anaemia is recognised in cardiac and vascular surgery. Anaemia is linked independently with worse outcome, including length of hospital stay and mortality. Despite studies having shown that intravenous iron is effective in a variety of population groups, ${ }^{11-14}$ there is still insufficient evidence to support its use. The current published evidence is of low quality or too heterogeneous. ${ }^{15}$ Furthermore, to our knowledge there are no RCTs that have been conducted looking at iron therapy in cardiovascular patients.

The UK CAVIAR Study is an observational, multicentre trial, to investigate the impact of anaemia and its management in cardiovascular patients, and the feasibility to study patients in the platform for a future planned RCT.
There is currently limited information on how iron treatment and major surgery have an influence on haematological parameters, functional parameters and patient outcome in cardiovascular patients. Hepcidin, a key regulator of iron homoeostasis ${ }^{9}$ was observed to be the only haematological variable that was associated with worse outcome in cardiac patients; and that nearly half of the patients identified had FID. ${ }^{8}$ Other studies that examined healthy participants and athletes with or without anaemia and iron deficiency had reduced functional capacity. ${ }^{28-30}$ It is therefore reasonable to assume that patients may respond to iron treatment, and may lead to improved outcomes. Our study is the first study to examine these areas and aims to provide insight on how intravenous iron and major surgery may influence hepcidin and other haematological variables, functional capacity and outcomes in cardiovascular patients. In addition, this study will also be the first to compare these two different, yet similar, population groups; and their outcomes assessed through using a composite of mortality and LOS in hospital (ie, DAOH).

One of the outcomes assessed in this study is patient $\mathrm{DAOH}$ at 30 days postsurgery. Traditionally in clinical trials, mortality and morbidity are often used as end point to determine treatment efficacy, however, it does not take into account the frequency for readmission, duration and severity. The DAOH is a composite score and is based on varying weightings placed on mortality and readmission factors, and therefore provides a clearer summary on the overall treatment effect. ${ }^{22}$

In our substudy, we are exploring tHbmass as an alternative marker in determining oxygen carrying capacity and in predicting postsurgical outcome. The insult of surgery puts enormous metabolic stress on the body and thereby an increase in the demand for oxygen is seen as a result. The inability to deliver enough oxygen to where it is needed may increase the risk of having poorer surgical outcomes. ${ }^{31} 32$ tHbmass represents absolute mass of circulating $\mathrm{Hb}$ and is relatively unexplored in the clinical setting, particularly in cardiovascular patients. Although $\mathrm{Hb}$ is traditionally used, studies have shown that $\mathrm{Hb}$ may not be a reliable or accurate measure as it can be influenced by fluid shifts (eg, blood loss) or haemodilution, which is commonly observed in ICU. ${ }^{26} 33$ If tHbmass provides a stronger link over $\mathrm{Hb}$ in predicting postsurgical outcome, this will be a useful measure in determining which and when patients can go into surgery; and can also be a target in raising tHbmass to improve outcome.

One of the key hurdles we anticipate in this study is the identification and recruitment of patients in the intravenous iron arm 10-42 days prior to surgery. The timeframe required is to allow at least 10 days for the intravenous iron to have an effect of increasing $\mathrm{Hb}$. As resources and infrastructure will vary between hospitals, this feasibility study will therefore help build the framework in setting up a preoperative anaemia pathway, and to best identify and manage these patients. 
Correcting anaemia preoperatively is recognised as an integral part of patient blood management by the NHS. Blood use in cardiac patients has been shown to increase every year, ${ }^{34}$ while vascular patients on average receive 2-3 units of blood during their hospital stay. ${ }^{35}$ The cost of transfusing a single unit of blood has been estimated to be $£ 170$ for the first unit, with the subsequent unit costing £162. If the number of units of blood used is reduced, there will be a potential saving of £146-689 per person. ${ }^{36}$ Apart from the cost-saving, the reduction in blood transfused in patients will reduce the likelihood of the associated worse outcomes that are often observed. These include postoperative infection and longer hospital stay. ${ }^{37}$ More importantly, for patients, correcting anaemia could significantly improve their outcome and quality of life as observed in patients with heart failure. $^{1114}$

The ultimate goal for this and the future RCTs is to have a preoperative anaemia pathway in place in every hospital so that patients with anaemia are identified early and corrected prior to surgery.

\section{Author affiliations}

${ }^{1}$ Division of Surgery \& Interventional Science, University College London, London, UK

${ }^{2}$ Cardiff \& Vale University Health Board, Cardiff, UK

${ }^{3}$ Department of Medical Statistics, London School of Hygiene \& Tropical Medicine, London, UK

${ }^{4}$ Department of Anaesthesia, Papworth Hospital, Cambridge, UK

Contributors TR and AK are the chief investigators, protocol authors and grant holders. $\mathrm{MC}$ is the manager for the trial and has drafted this manuscript. CE assisted in the design of the study and helped draft the manuscript. $A B$ assisted in drafting the manuscript. TC participated in the statistical design of the study. All authors read and approved the final manuscript.

Funding This project was funded by Pharmacosmos A/S, and the National Institute of Academic Anaesthesia (NIAA) - jointly funded by Association of Cardiothoracic Anaesthetists (ACTA), the Vascular Anaesthesia Society of Great Britain and Ireland (VASGBI), and British Journal of Anaesthesia (BJA)/Royal College of Anaesthetists (RCOA).

Competing interests MC's salary is supported by Pharmacosmos A/S. TR reports grants from NIHR HTA (UK); grants from NHMRC (Australia); grants from NIAA / BJA / ACTA / VASGBI (UK); grants, personal fees and non-financial support from Pharmacosmos; grants, personal fees and non-financial support from Vifor Pharma; grants, personal fees and non-financial support from Acelity; grants, personal fees and non-financial support from Stroke Association; grants from Mason Medical Research Foundation; grants from UCH League of Friends; grants and non-financial support from Libresse/ Bodyform; outside the submitted work. TR is a regular speaker at national and international conferences on anaemia, blood transfusion, wound healing and vascular diseases for which he has received expenses for travel, accommodation and sundries. TR is the Director of The IronClinic Ltd, Director of Veincare London Ltd, and is also the Vascular lead for 18-week wait Ltd. CE is a Consultant for Pharmacosmos. AK has received research funding and honoraria from Pharmacosmos, Vifor Pharma, CSL Behring and Brightwake Ltd. AK is the Editor-in-Chief of Anaesthesia. There is no external or industry involvement in CAVIAR.

Ethics approval London Westminster Research Ethics Committee.

Provenance and peer review Not commissioned; externally peer reviewed.

Open Access This is an Open Access article distributed in accordance with the Creative Commons Attribution Non Commercial (CC BY-NC 4.0) license, which permits others to distribute, remix, adapt, build upon this work non-commercially, and license their derivative works on different terms, provided the original work is properly cited and the use is non-commercial. See: http://creativecommons.org/ licenses/by-nc/4.0/ (c) Article author(s) (or their employer(s) unless otherwise stated in the text of the article) 2017. All rights reserved. No commercial use is permitted unless otherwise expressly granted.

\section{REFERENCES}

1. Ranucci M, Baryshnikova E, Castelvecchio S, et al; Surgical and Clinical Outcome Research (SCORE) Group. Major bleeding, transfusions, and anemia: the deadly triad of cardiac surgery. Ann Thorac Surg 2013;96:478-85.

2. Hung M, Besser M, Sharples LD, et al. The prevalence and association with transfusion, intensive care unit stay and mortality of pre-operative anaemia in a cohort of cardiac surgery patients. Anaesthesia 2011;66:812-8.

3. Baron DM, Hochrieser H, Posch M, et al; European Surgical Outcomes Study (EuSOS) group for Trials Groups of European Society of Intensive Care Medicine, European Society of Anaesthesiology. Preoperative anaemia is associated with poor clinical outcome in non-cardiac surgery patients. Br J Anaesth 2014;113:416-23.

4. Kulier A, Levin J, Moser R, et al; Investigators of the Multicenter Study of Perioperative Ischemia Research Group, Ischemia Research and Education Foundation. Impact of preoperative anemia on outcome in patients undergoing coronary artery bypass graft surgery. Circulation 2007;116:471-9.

5. Musallam KM, Tamim HM, Richards T, et al. Preoperative anaemia and postoperative outcomes in non-cardiac surgery: a retrospective cohort study. Lancet 2011;378:1396-407.

6. Gaskell H, Derry S, Andrew Moore R, et al. Prevalence of anaemia in older persons: systematic review. BMC Geriatr 2008;8:1.

7. Klein AA, Collier TJ, Brar MS, et al; Association of Cardiothoracic Anaesthetists (ACTA). The incidence and importance of anaemia in patients undergoing cardiac surgery in the UK - the first association of cardiothoracic anaesthetists national audit. Anaesthesia 2016;71:627-35.

8. Hung M, Ortmann E, Besser M, et al. A prospective observational cohort study to identify the causes of anaemia and association with outcome in cardiac surgical patients. Heart 2015;101:107-12.

9. D'Angelo G. Role of hepcidin in the pathophysiology and diagnosis of anemia. Blood Res 2013;48:10-15.

10. Weiss G, Goodnough LT. Anemia of chronic disease. N Engl J Med 2005;352:1011-23.

11. Bolger AP, Bartlett FR, Penston HS, et al. Intravenous iron alone for the treatment of anemia in patients with chronic heart failure. J Am Coll Cardiol 2006;48:1225-7.

12. Schröder O, Mickisch $O$, Seidler U, et al. Intravenous iron sucrose versus oral iron supplementation for the treatment of iron deficiency anemia in patients with inflammatory bowel disease--a randomized, controlled, open-label, multicenter study. Am J Gastroenterol 2005;100:2503-9.

13. Theusinger OM, Leyvraz PF, Schanz U, et al. Treatment of iron deficiency anemia in orthopedic surgery with intravenous iron: efficacy and limits: a prospective study. Anesthesiology 2007;107:923-7.

14. Anker SD, Comin Colet J, Filippatos G, et al; FAIR-HF Trial Investigators. Ferric carboxymaltose in patients with heart failure and iron deficiency. N Engl J Med 2009;361:2436-48.

15. Gurusamy KS, Nagendran M, Broadhurst JF, et al. Iron therapy in anaemic adults without chronic kidney disease. Cochrane Database Syst Rev 2014;12:CD010640.

16. Clevenger B, Gurusamy K, Klein AA, et al. Systematic review and meta-analysis of iron therapy in anaemic adults without chronic kidney disease: updated and abridged cochrane review. Eur J Heart Fail 2016;18:774-85.

17. Hogan M, Klein AA, Richards T. The impact of anaemia and intravenous iron replacement therapy on outcomes in cardiac surgery. Eur J Cardiothorac Surg 2015;47:218-26.

18. National institute for health and care excellence guideline. Blood transfusion 2015. https://www.nice.org.uk/guidance/ng24

19. Mann CJ. Observational research methods. research design II: cohort, cross sectional, and case-control studies. Emerg Med J 2003;20:54-60.

20. Organisation WH. WHO). Haemoglobin concentrations for the diagnosis of anaemia and assessment of severity 2011. http://www. who.int/vmnis/indicators/haemoglobin.pdf

21. Blanc B, Finch C, Hallberg L, et al; Nutritional Anaemias. World Health Organ Tech Rep Ser 1968;405:5-37.

22. Ariti CA, Cleland JG, Pocock SJ, et al. Days alive and out of hospital and the patient journey in patients with heart failure: insights from the 
candesartan in heart failure: assessment of reduction in mortality and morbidity (CHARM) program. Am Heart J 2011;162:900-6.

23. Agnew N. Preoperative cardiopulmonary exercise testing. Continuing Education in Anaesthesia, Critical Care \& Pain 2010;10:33-7.

24. ATS Committee on Proficiency Standards for Clinical Pulmonary Function Laboratories. ATS statement: guidelines for the six-minute walk test. Am J Respir Crit Care Med 2002;166:111-7.

25. Schmidt W, Prommer N. The optimised CO-rebreathing method: a new tool to determine total haemoglobin mass routinely. Eur J App Physiol 2005;95:486-95.

26. Otto JM, Montgomery HE, Richards T. Haemoglobin concentration and mass as determinants of exercise performance and of surgical outcome. Extrem Physiol Med 2013;2:33.

27. NHS blood and transplant. National Comparative Audit of Blood Transfusion 2015. http://hospital.blood.co.uk/media/28123/2015pbm-in-scheduled-surgery-audit-report.pdf

28. Celsing F, Blomstrand E, Werner B, et al. Effects of iron deficiency on endurance and muscle enzyme activity in man. Med Sci Sports Exerc 1986;18:156-61.

29. Klingshirn LA, Pate RR, Bourque SP, et al. Effect of iron supplementation on endurance capacity in iron-depleted female runners. Med Sci Sports Exerc 1992;24:819-24.
30. Newhouse IJ, Clement DB, Taunton JE, et al. The effects of prelatent/ latent iron deficiency on physical work capacity. Med Sci Sports Exerc 1989;21:263-8.

31. Peerless JR, Alexander JJ, Pinchak AC, et al. Oxygen delivery is an important predictor of outcome in patients with ruptured abdominal aortic aneurysms. Ann Surg 1998;227:726-34.

32. Sanders J, Toor IS, Yurik TM, et al. Tissue oxygen saturation and outcome after cardiac surgery. Am J Crit Care 2011;20:138-45.

33. Astin R, Puthucheary $Z$. Anaemia secondary to critical illness: an unexplained phenomenon. Extrem Physiol Med 2014;3:4.

34. Goodnough LT. Risks of blood transfusion. Anesthesiol Clin North America 2005;23:241-52.

35. Menis M, Anderson SA, Forshee RA, et al. Transfusion-related acute lung injury and potential risk factors among the inpatient US elderly as recorded in medicare claims data, during 2007 through 2011. Transfusion 2014;54:2182-93.

36. National Institute for Health and Care Excellence. Costing statement: Blood transfusion 2015. https://www.nice.org.uk/ guidance/ng24

37. Gombotz H, Rehak PH, Shander A, et al. Blood use in elective surgery: the Austrian benchmark study. Transfusion 2007;47:1468-80. 